\title{
Quantitative Online Liquid Chromatography-Surface-Enhanced Raman Scattering of Purine Bases
}

\author{
David P. Cowcher, Roger Jarvis, and Royston Goodacre* \\ School of Chemistry and Manchester Institute of Biotechnology, University of Manchester, 131 Princess Street, Manchester, M1 \\ 7DN, U.K.
}

\section{Supporting Information}

\begin{abstract}
Raman spectroscopy has been of interest as a detection method for liquid chromatographic separations for a significant period of time, due to the structural information it can provide, allowing the identification and distinction of coeluting analytes. Combined with the rapidly advancing field of enhanced Raman techniques, such as surface-enhanced Raman scattering (SERS), the previous low sensitivity of Raman measurements has also been alleviated. At-line LCSERS analyses, where SERS measurements are taken of

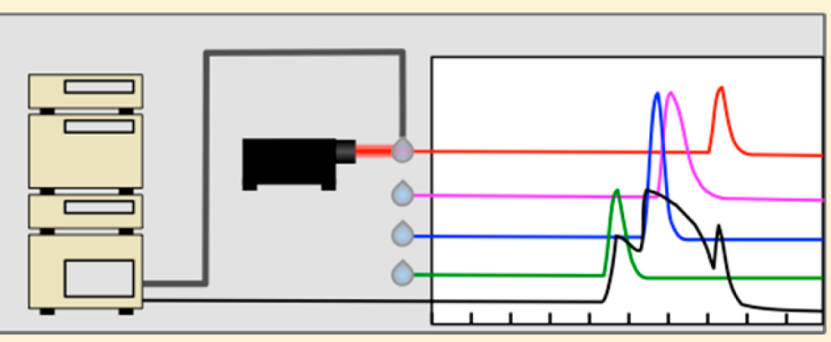
fractions collected during or after HPLC separation have been shown to be sensitive and applicable to a wide variety of analytes; however, quantitative, real-time, online LC-SERS analysis at comparable sensitivity to existing methods, applicable to high-throughput experiments, has not been previously demonstrated. Here we show that by introducing silver colloid, followed by an aggregating agent into the postcolumn flow of an HPLC system, we can quantitatively and reproducibly analyze mixtures of purine bases, with limits of detection in the region of 100-500 pmol. The analysis is performed without the use of a flow cell, thereby eliminating previously detrimental memory effects.
\end{abstract}

$\mathrm{V}$ ibrational spectroscopic techniques such as infrared (IR) ${ }^{1}$ and Raman ${ }^{2-6}$ are emerging as useful, novel detectors for high-performance liquid chromatography (HPLC) analyses, as they can provide detailed structural information, allowing definitive analyte identification. Furthermore, their analyses are rapid and the instrumentation is relatively small and inexpensive compared to techniques such as mass spectrometry (MS) and NMR, making online analysis a realistic possibility. The development of vibrational spectroscopic detection for high-pressure liquid chromatography (HPLC) has thus far not progressed at the speed of other techniques due to the fact that many of the solvents required for the mobile phase, particularly in reversed-phase HPLC (RP-HPLC), give a strong background signal, often masking the analytes of interest. However, with recent advances in data processing techniques, solvent backgrounds are able to be more effectively removed. ${ }^{1}$

A particularly exciting area of vibrational spectroscopy for many applications where low analyte levels are found is surfaceenhanced Raman scattering (SERS). This approach provides vastly superior sensitivity compared to conventional Raman scattering, it retains structural and chemical information, and provides the benefit over IR of minimal interference from water vibrations. SERS uses predominantly metal surfaces with nanoscale features to enhance the incident and scattered light in their vicinity, thereby increasing the detected signal by many orders of magnitude.

The combination of HPLC and SERS detection is not new and various examples have been reported previously. ${ }^{4}$ These can be divided into two main groups: online $e^{2,8,9}$ and atline. ${ }^{5,6,10-12}$ The former interfaces SERS detection directly with the HPLC system, meaning analysis is conducted in real time. While this approach is ideal in that it allows high-throughput analysis and data collection that takes no longer than the chromatographic run itself, there are inherent difficulties. For example, with colloid-based SERS approaches, the nanoparticles need to be introduced into the mobile phase flow and the colloid allowed to mix and aggregate sufficiently for a maximum SERS response to be achieved, while at the same time minimizing the distance between column and detector to prevent sample diffusion and loss of analyte resolution. There are also issues with memory effects: the colloid sticking to the inside of flow cells used to collect online SERS spectra, thus requiring cleaning between samples. A nice idea incorporating a windowless flow cell was presented by Cabalin et al. ${ }^{9,13}$ but their reported limits of detection (LOD) were significantly higher than UV absorbance detection. Likewise with fixed substrate-based SERS detection, problems arise in the cleaning of the substrate to ensure that a reproducible SERS response is obtained from sample to sample. These memory effects were the main reason why online HPLC-SERS using roughened electrodes has ceased. ${ }^{4}$

At-line SERS detection deals with the separation and detection parts of the analysis separately, with the separation being completed first and effluent fractions collected throughout the run. These fractions are then analyzed off-line

Received: August 4, 2014

Accepted: September 6, 2014

Published: September 7, 2014 
using a separate Raman instrument. As there is no longer a time-critical element to the analysis, care can be taken to optimize the SERS conditions for each fraction independently and there is no need to compromise SERS response for separation efficiency, as is often the case with online methods. This does mean of course that at-line methods are so far nowhere near as high-throughput as online ones.

Analysis of purine bases forms a large part of the existing literature on SERS detection for HPLC and coupled with the fact that there is a wealth of literature on purine detection by other techniques (just a few relevant examples cited), including UV absorbance, ${ }^{14-16}$ UV diode array, ${ }^{17}$ mass spectrometry, ${ }^{18-21}$ and electrochemical methods ${ }^{22,23}$ from real-life samples $^{24,25}$ as well as synthetic mixtures, suggested this was a sensible place to begin our investigation. The purine bases adenine and guanine are constituents of both DNA and RNA. They are found in tissues, cells, and physiological fluids as a result of nucleic acid catabolism, tissue degradation, diet, and nucleotide salvage. ${ }^{2}$ They have also been shown to be susceptible to deamination by reactive nitrogen species in the body to form the breakdown products hypoxanthine and xanthine. ${ }^{26}$ The accurate quantification of these purine bases, together with their metabolites, is valuable, as differences in the amounts present in excreted fluids such as urine have been detected between healthy and nonhealthy patients with various cancers. $^{27,28}$ There are also industrial applications, such as food shelf life determination. ${ }^{29}$

As mentioned previously, analysis of purine bases and their derivatives by RP-HPLC is not new and there are many previously published methods; however, the most commonly used detection method was UV absorbance, ${ }^{30}$ and in order to obtain suitable resolution of different purines, sophisticated chromatography is required; for example, by the inclusion of ion-pairing species $^{31}$ and even then, definitive identification from a complex mixture based on retention time alone is likely to prove problematic. In order to overcome these chemical species' resolution difficulties, HPLC has been coupled with detection techniques that can provide more detailed analyte identification; most popular are mass spectrometry ${ }^{18,20,32-35}$ and nuclear magnetic resonance (NMR) spectroscopy. ${ }^{35,36}$

Both online ${ }^{2}$ and at-line ${ }^{5}$ SERS detection has been demonstrated for purine base separations. The former used a heated colloid as the SERS substrate, which was introduced directly into the mobile phase flow postcolumn. Thermal aggregation was used to increase the SERS intensity but quantitative detection was not possible. The slower latter method was able to quantify a mixture of purine and pyrimidine bases using micro-LC separation, followed by fraction collection in microtiter plates. Silver quantum dots were then added to these fractions and the $\mathrm{pH}$ adjusted to provide optimum SERS conditions before analysis with a relatively expensive and bulky confocal Raman microscope. While this atline work ${ }^{5}$ provides a very robust analysis, incorporating a high level of control, the extra time required for analysis of each sample will significantly affect its applicability to high throughput experiments and the large sample sets often encountered with metabolomics experiments. ${ }^{37}$

In this study we have shown for the first time, to the best of our knowledge, that through introducing a separate aggregating agent into the mobile phase flow after the silver colloid allows for a fully quantitative, online analysis of mixed analytes. By removing the traditional flow cell altogether, we can also eliminate the memory effects that have plagued previous online
SERS attempts. Using purine base samples as an example, even when the HPLC peaks are coeluting, it is possible to use a simple portable Raman spectrometer to generate data that rival traditional UV detection, and the developed LC-SERS method has much lower limits of detection.

\section{EXPERIMENTAL SECTION}

Reagents and Materials. Adenine (99\%), guanine (99\%), hypoxanthine $(\geq 99 \%)$, xanthine $(\geq 99 \%)$, acetic acid (LCMSgrade), and trisodium citrate (analytical grade) were all used as obtained from Sigma-Aldrich Ltd. (Dorset, U.K.). Potassium dihydrogen phosphate and silver nitrate (both analytical grade) were used as obtained from Fisher Scientific (Loughborough, U.K.) Phosphoric acid (85\%) and potassium nitrate (99\%) were used as obtained from Alfa Aesar (Heysham, U.K.). Perchloric acid (60\%) was used as obtained from BDH Ltd., (Poole, U.K.). All water used for this work was of HPLC purity and supplied by Sigma-Aldrich (Dorset, U.K.) unless otherwise stated.

Nanoparticle Synthesis. Citrate-reduced silver colloid was synthesized according to the method described by Lee and $\mathrm{Meisel}^{38}$ in $1.5 \mathrm{~L}$ batches. All glassware was precleaned using aqua regia and washed thoroughly with deionized water. A mass of $270 \mathrm{mg}$ of silver nitrate was dissolved in $1500 \mathrm{~mL}$ of water and heated to boiling. A volume of $30.0 \mathrm{~mL}$ of $1 \%$ weight/ volume $(\mathrm{w} / \mathrm{v})$ trisodium citrate in water was added dropwise with vigorous stirring, and the mixture was left to boil for $\sim 1 \mathrm{~h}$. The gradual appearance of a green-gray suspension indicated successful nanoparticle formation. After $\sim 1 \mathrm{~h}$, the nanoparticle suspension was allowed to cool and stored protected from light. The nanoparticle size distribution was characterized by UV-vis spectrophotometry to allow comparison of different batches and was very similar to colloid prepared by us previously. ${ }^{39,40}$ The colloid was agitated before use to ensure any settled particles were resuspended.

Sample Preparation. The $1.00 \mathrm{mM}$ stock solutions of the purine bases were prepared according to the method described by Carrillo-Carrión et al. ${ }^{5}$ Adenine was dissolved in water and guanine, hypoxanthine and xanthine in $2.00 \mathrm{M}$ perchloric acid. Samples for individual analysis were then prepared by diluting the stock solutions with the appropriate amount of $0.010 \mathrm{M} \mathrm{pH}$ 3.0 potassium phosphate buffer. Stock solutions for mixture analysis were prepared in the same way at four times the concentration required in the final mixture for each analyte $\left(4.00 \times 10^{-4} \mathrm{M}, 2.00 \times 10^{-4} \mathrm{M}, 1.00 \times 10^{-4} \mathrm{M}, 4.00 \times 10^{-5} \mathrm{M}\right.$, and $\left.2.00 \times 10^{-5} \mathrm{M}\right)$. Equal aliquots of each of the relevant stocks were then mixed together (four per mixture), giving the desired final concentrations.

Instrumentation. HPLC separation was conducted using a Shimadzu LC-10AVP HPLC system (Kyoto, Japan), consisting of an online degasser, two mobile phase pumps, autosampler, column oven, and variable wavelength UV absorbance detector.

SERS analysis was performed using a small DeltaNu Advantage 200A portable Raman spectrometer (Laramie, WY) with $633 \mathrm{~nm}$ laser excitation. An optical extension tube was fitted to the front of the spectrometer in place of the usual sample holder to allow external Raman excitation with a power of $3 \mathrm{~mW}$ at the sample. Scattered light was collected by the same aperture at $180^{\circ}$ to the excitation direction. Colloid for SERS analysis was introduced from a syringe using a syringe driver (KD Scientific, Holliston, MA).

The HPLC system itself was unaltered and operated in the standard UV absorbance detection mode. All modifications to 
the solvent flow occurred downstream of the UV detector. Silver nanoparticles were introduced to the mobile phase from a $60 \mathrm{~mL}$ syringe on a syringe driver, by means of a T-mixer. Immediately downstream of this mixer, $0.500 \mathrm{M}$ potassium nitrate aggregating agent was introduced from a second HPLC mobile phase pump by means of a second T-mixer. Following the introduction of the colloid and aggregating agent, the HPLC tubing ended in front of the aperture of the Raman spectrometer, where the mobile phase/colloid/aggregating agent mixture dripped from the tube as Raman spectra were recorded of the forming droplets. The position of the tube end relative to the spectrometer aperture was optimized by collecting $1 \mathrm{~s}$ spectra in continuous mode until the signal response was maximized. A diagram of the instrument setup is shown in Figure 1, and pictures of the apparatus are provided in Figure S1 in the Supporting Information.

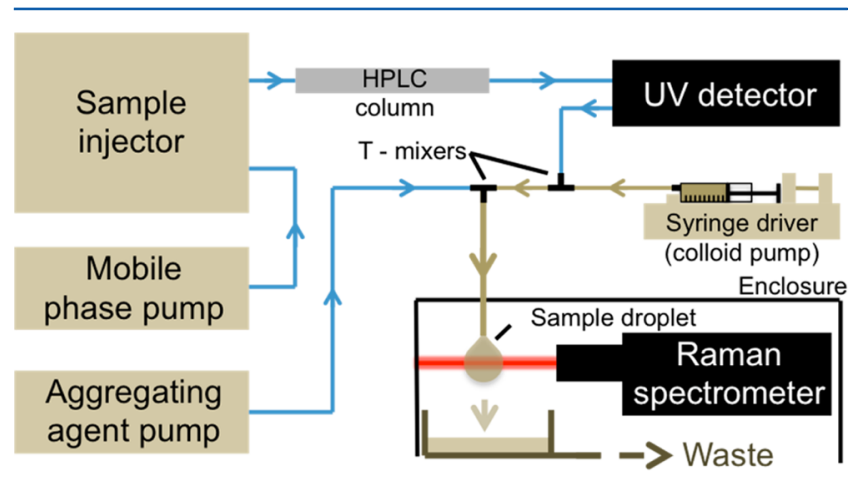

Figure 1. Schematic diagram of the LC-SERS instrumentation.

Sample Analysis. HPLC separation was conducted using a $150 \mathrm{~mm} \times 4.6 \mathrm{~mm}, 3 \mu \mathrm{m}$ particle size, Supelcosil LC-18-T $\mathrm{C}_{18}$ column (Supelco, Bellefonte, PA), fitted with a column guard and maintained at $25{ }^{\circ} \mathrm{C}$ in a column oven. For each injection, the run time was $8.0 \mathrm{~min}$. The mobile phase was $0.010 \mathrm{M}$ aqueous potassium phosphate buffer at $\mathrm{pH}$ 3.0, pumped at a flow rate of $1.0 \mathrm{~mL} \mathrm{~min}{ }^{-1}$. A volume of $50 \mu \mathrm{L}$ of each sample was introduced using an autoinjector. UV absorbance detection was measured at $260 \mathrm{~nm}$, as determined from UV-vis spectra of the individual analytes (data not shown).

Silver colloid for SERS measurements was introduced at 1.0 $\mathrm{mL} \min ^{-1}$ and the potassium nitrate aggregating agent at 0.2 $\mathrm{mL} \min ^{-1}$. There was sufficient time to change over the colloid syringe between the end of one sample's analysis and the injection of the next. This did not affect the SERS data, meaning this was not a limiting factor in the length of a sample batch. SERS spectra were recorded using the multiacquire mode of the DeltaNu spectrometer, with an individual integration time of $1 \mathrm{~s}$.

Data Analysis. All data were exported from the respective instruments' operating software and analyzed using Matlab R2013a (The Mathworks, Natick, MA). No preprocessing was conducted on the UV absorbance data. In order to remove the background citrate peaks, SERS spectra were row normalized against their total signal and then normalized against the mean SERS spectrum from a sample injection of just the potassium phosphate buffer. The code for these preprocessing steps was written in-house and is available on request.

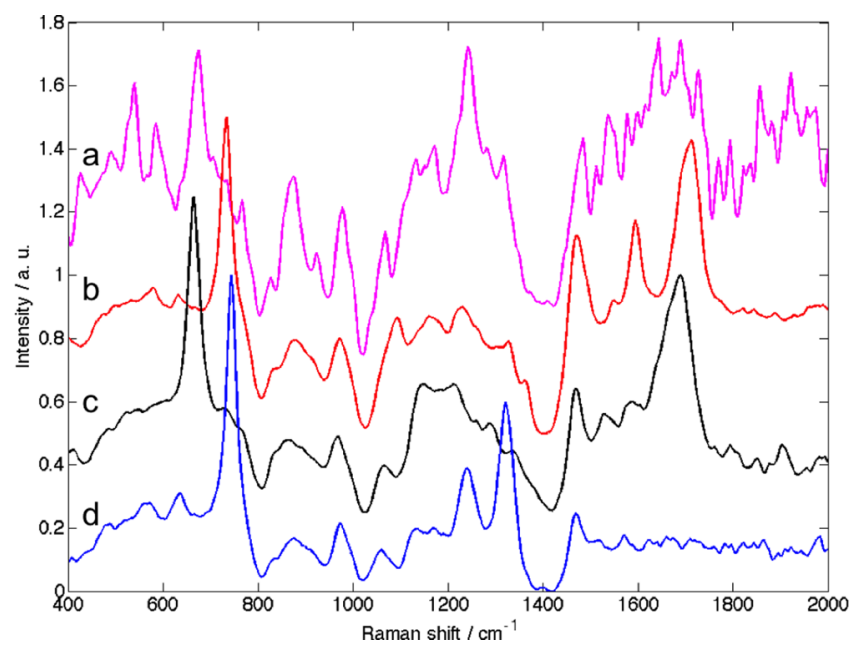

Figure 2. SERS spectra of the four purine bases at their peak retention times in the range 400-2000 $\mathrm{cm}^{-1}$. (a) Xanthine, (b) hypoxanthine, (c) guanine, and (d) adenine. The spectra here are preprocessed as they would be for analysis: normalized against total signal and against the mean blank spectrum. To provide a better visual comparison, these examples have been offset and have also been scaled on the $y$-axis between 0 and 1 .

\section{RESULTS AND DISCUSSION}

The analyte solvent and mobile phase were prepared at $\mathrm{pH} 3.0$ in order to ensure the purine bases remained preferentially in their lactam form, ${ }^{2}$ which reduces the effect of vertical base stacking. ${ }^{30}$ As such, the four components eluted from the HPLC column within 8 min.

In previous nanoparticle-based SERS experiments, for example, bacterial spore detection, ${ }^{40}$ we have found that mixing equal volumes of sample and colloid gives good SERS spectra. Therefore, Ag colloid was pumped at the same flow rate as the HPLC mobile phase $\left(1 \mathrm{~mL} \mathrm{~min}^{-1}\right)$, and this was found to provide good SERS data via preliminary experimentation (data not shown). A syringe driver was chosen to introduce the silver colloid to the system, as the use of more sophisticated liquid delivery instruments would risk lasting contamination and damage to their internal workings through deposition of nanoparticles. The SERS detector was set up downstream to a UV absorbance detector so that the SERS data could be directly compared to the UV data from the same samples, although once a method has been validated using SERS detection the UV absorbance detector could be omitted to minimize postcolumn analyte diffusion.

In order to test the extent of the colloid memory effect with online aggregation, ${ }^{4}$ the SERS detector was initially set up with a quartz capillary tube passing though the conventional sample holder of the DeltaNu instrument. This allowed successful collection of SERS data along with straightforward alignment; however, the internal walls of the capillary tube quickly developed a coating of silver as reported previously; a photo of which is shown in Figure S2 in the Supporting Information. Therefore, the quartz capillary was removed and the sample holder replaced with an optical extension tube that allows analysis of samples external to the Raman instrument. The end of the HPLC tubing was positioned just above the Raman aperture so that as a droplet of the sample formed, it was optimally within in the focus of the spectrometer.

The use of a liquid drop as an optical cell has previously been described, ${ }^{41}$ but to the best of our knowledge this is the first 
Table 1. Metrics for HPLC Analysis of Individual Nucleobase Samples Using Simultaneous UV and SERS Detection

\begin{tabular}{|c|c|c|c|c|c|}
\hline & analyte & adenine & guanine & hypoxanthine & xanthine \\
\hline & retention time/s & 268 & 226 & 254 & 311 \\
\hline \multirow[t]{8}{*}{ UV } & linearity & 0.9841 & 0.9892 & 0.9935 & 0.9839 \\
\hline & $\mathrm{LOD}($ concn $) / \mathrm{M}$ & $4.25 \times 10^{-6}$ & $2.97 \times 10^{-6}$ & $1.95 \times 10^{-6}$ & $2.79 \times 10^{-6}$ \\
\hline & LOD (on column) $/ \mathrm{mol}$ & $2.13 \times 10^{-10}$ & $1.49 \times 10^{-10}$ & $9.76 \times 10^{-11}$ & $1.40 \times 10^{-10}$ \\
\hline & $\mathrm{LOQ}($ concn $) / \mathrm{M}$ & $7.37 \times 10^{-6}$ & $7.62 \times 10^{-6}$ & $3.45 \times 10^{-6}$ & $6.13 \times 10^{-6}$ \\
\hline & LOQ (on column) $/ \mathrm{mol}$ & $3.69 \times 10^{-10}$ & $3.81 \times 10^{-10}$ & $1.72 \times 10^{-10}$ & $3.06 \times 10^{-10}$ \\
\hline & mean linearity of leave-one-out calibration & 0.9839 & 0.9891 & 0.9934 & 0.9838 \\
\hline & linearity of leave-one-out predicted vs actual concn & 0.9812 & 0.987 & 0.9916 & 0.9779 \\
\hline & RMS error of leave-one-out predicted vs actual concn/M & $2.27 \times 10^{-6}$ & $1.87 \times 10^{-6}$ & $1.50 \times 10^{-6}$ & $2.48 \times 10^{-6}$ \\
\hline \multirow[t]{10}{*}{ SERS } & peak position $/ \mathrm{cm}^{-1}$ & 744 & 666 & 734 & 672 \\
\hline & peak assignment & \multicolumn{4}{|c|}{ ring breathing ${ }^{22}$} \\
\hline & linearity & 0.9816 & 0.9976 & 0.992 & 0.9863 \\
\hline & $\operatorname{LOD}($ concn $) / \mathrm{M}$ & $1.58 \times 10^{-6}$ & $9.38 \times 10^{-7}$ & $1.16 \times 10^{-6}$ & $1.98 \times 10^{-6}$ \\
\hline & $\mathrm{LOD}($ on column $) / \mathrm{mol}$ & $7.88 \times 10^{-11}$ & $4.68 \times 10^{-11}$ & $5.79 \times 10^{-11}$ & $9.86 \times 10^{-11}$ \\
\hline & LOQ $($ concn $) / M$ & $2.26 \times 10^{-6}$ & $1.79 \times 10^{-6}$ & $4.40 \times 10^{-6}$ & $3.39 \times 10^{-6}$ \\
\hline & $\mathrm{LOQ}$ (on column) $/ \mathrm{mol}$ & $1.13 \times 10^{-10}$ & $8.9 \times 10^{-11}$ & $2.20 \times 10^{-10}$ & $1.70 \times 10^{-10}$ \\
\hline & mean linearity of leave-one-out calibration & 0.9821 & 0.9976 & 0.995 & 0.9862 \\
\hline & linearity of leave-one-outpredicted vs actual concn & 0.9624 & 0.9962 & 0.9933 & 0.9822 \\
\hline & RMS error of leave-one-out predicted vs actual concn/M & $3.26 \times 10^{-6}$ & $1.01 \times 10^{-6}$ & $1.34 \times 10^{-6}$ & $2.20 \times 10^{-6}$ \\
\hline
\end{tabular}

time it has been applied to online HPLC-SERS analysis. The precise position of this tubing was optimized by pumping the HPLC mobile phase, colloid, and aggregating agent and observing the SERS response of the citrate background, with the instrument in continuous mode (Photographs of this setup are show in Figure S1 in the Supporting Information). While the short length of tubing between the aggregating agent $\mathrm{T}$ mixer and spectrometer is technically able to experience colloid memory effects, this was not observed during any of the sample analyses. Replicate samples were not analyzed sequentially in order to make any differences from memory effects showing up in the data.

The potassium nitrate aggregating agent was introduced at $0.2 \mathrm{~mL} \mathrm{~min}{ }^{-1}$, which means that at a concentration of $0.5 \mathrm{M}$ there is more aggregating agent present than would normally be necessary for a good SERS measurement; however, the excess ensures that aggregation occurs rapidly, producing optimum SERS conditions by the time the mixture drips in front of the Raman aperture. The typical time from aggregate addition to measurement is $>1 \mathrm{~s}$.

During sample analysis, sequential Raman spectra were recorded with a $1.0 \mathrm{~s}$ integration time, using the instrument's "multiacquire" setting. With the combined flow rate of $2.2 \mathrm{~mL}$ $\mathrm{min}^{-1}$, multiple individual droplets pass in front of the Raman aperture during the collection of each spectrum, thereby allowing a small level of eluent averaging. Ideally the instrument would begin recording the next spectrum as soon as it had finished the previous one, but while the individual spectra are only recorded for $1.0 \mathrm{~s}$, the current instrument takes time to save the data file and move onto the next spectrum. As a result of this, spectra were only able to be recorded approximately every $7.5 \mathrm{~s}$. While this is far from optimal, the sampling rates could be improved with modifications to the instrument and its operating software. The retention time axis was constructed accurately by dividing the total number of measurements recorded during each injection by the total run time.

The chromatographic conditions were deliberately kept straightforward, with a single-component, isocratic mobile phase. This avoided the requirement for additional ion-pairing reagents or organic modifiers that might interfere with the
SERS detection. Unfortunately this increased peak tailing, particularly with adenine (vide infra), but this compromise was made in order to allow us to demonstrate that the SERS spectra can be used to quantify coeluting peaks of different analytes. While we chose to keep our elution method as simple as possible using isocratic elution, this does not necessarily mean that organic modifiers and gradient elution methods are unsuitable for use with SERS detection. The biggest problem, one that has historically slowed the advancement of vibrational spectroscopic detection for HPLC, would be likely to come from the fact that organic solvents used in gradient elution tend to be strong Raman scatterers, thereby possessing the potential to mask any analyte peaks; although steps such as those described below for the removal of the citrate spectra may significantly improve the situation. In addition, if the organic component had an affinity for the silver nanoparticles, the competition with the analyte and citrate capping agent at the nanoparticle surface would need to be accounted for. While it would be sensible to employ organic modifiers that are selected carefully to try and reduce complex competitive interactions, this would be achieved by running analyte standards through the gradient elution setup and plotting responses versus concentration and using suitable curve fitting to effect quantification.

SERS spectra throughout the run are dominated by peaks from the citrate capping agent of the colloid. This background was very intense and highly reproducible, with intensity fluctuations only coming from the lensing affect of the liquid droplets forming and falling in front of the spectrometer. However, when analyte(s) was present, the citrate was readily replaced at the surface of the colloid as evident by the citrate signal intensity dropping off significantly. This provided us with the advantage that we were able to isolate the analyte signal very effectively, first by normalizing each spectrum against its total signal (row-normalization) and then by dividing each spectrum by the mean row-normalized spectrum of a blank sample from the same chromatographic run, as shown in Figure S3 in the Supporting Information. A similar method has previously been described by Quintás et al. ${ }^{42}$ for HPLC-IR spectroscopic data. 

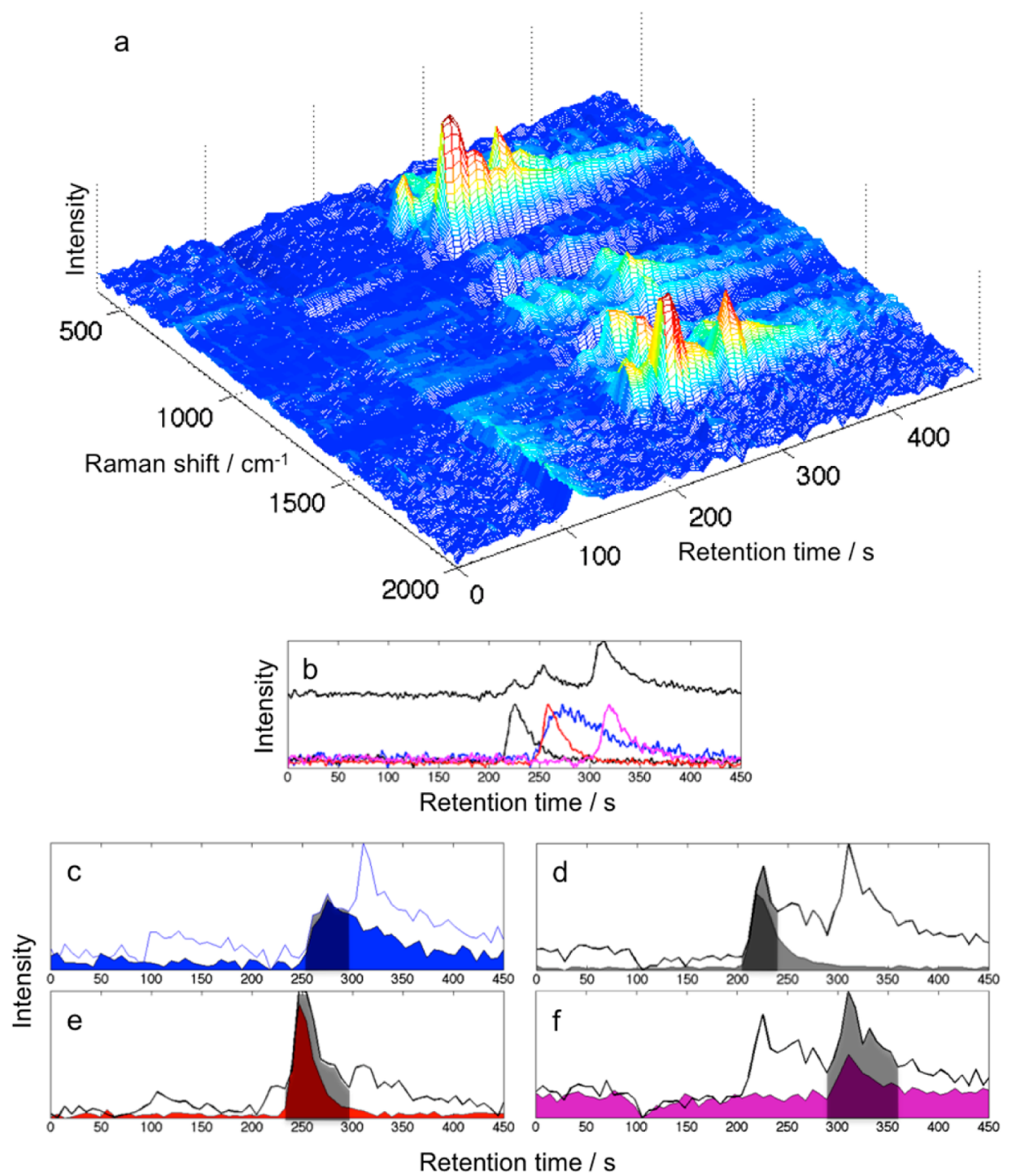

Figure 3. Example LC-SERS data from a single injection of a sample containing $2.5 \times 10^{-5} \mathrm{M}$ guanine, $5 \times 10^{-5} \mathrm{M}$ hypoxanthine, $1 \times 10^{-5} \mathrm{M}$ adenine, and $1 \times 10^{-4} \mathrm{M}$ xanthine, eluted in that order. Each SERS spectrum throughout the data collection has been normalized against its total signal and against the mean blank spectrum. Part a shows the 3D LC-SERS data plot for the spectral region between $400-2000 \mathrm{~cm}^{-1}$. Part b shows the corresponding UV chromatogram (top) along with scaled UV chromatograms from injections of the individual analytes below. Parts $\mathrm{c}-\mathrm{f}$ show SERS intensity with respect to retention time (line), with scaled SERS chromatograms from injections of the individual analytes (solid color) and the area measured for quantification (shaded) for (c) adenine at $1322 \mathrm{~cm}^{-1}$, (d) guanine at $669 \mathrm{~cm}^{-1}$, (e) hypoxanthine at $1713 \mathrm{~cm}^{-1}$, and (f) xanthine at $678 \mathrm{~cm}^{-1}$.

Following these data processing steps, example spectra of the four individual purine bases are shown in Figure 2. Using vibrations specific to each of the analytes, their theoretical limit of detection (LOD) and limit of quantification (LOQ), as well as the reproducibility using UV absorbance and SERS, were determined simultaneously by repeatedly injecting three replicates of individual purine base samples at varying concentrations. In addition, a leave-one-out (LOO) analysis was performed, in which a single data point was removed from the data set and a calibration model (again from individual analyte-specific SERS peaks) developed based on the remaining data. This model was then used to predict the concentration of the data point that was left out. The whole process was repeated for each individual data point in turn. These results are summarized in Table 1, with the associated calibration plots and plots of the leave-one-out predictions shown in Figures S4-S7 in the Supporting Information. LODs are calculated from the mean blank value plus 3 times its standard deviation and the LOQs from the mean blank value plus 10 times its standard deviation.

The SERS data showed excellent quantitative behavior, with better linearity and lower LOD/LOQ than the UV absorbance detection for each analyte. The LOO analysis was also better for the SERS data than the UV in most cases, with just the adenine results marginally worse in the SERS. However, this was affected by a single anomalous result at the high extreme of the adenine calibration that is likely to be due to the nonideal peak tailing seen for adenine as a result of the chromatographic conditions chosen, which suggests overloading of this analyte. The inset of the adenine plot in Figure S7 in the Supporting Information shows that at lower concentration the predicted results are very accurate and reproducible.

Mixed-analyte samples were then analyzed under the same conditions and following the same data processing steps. An 

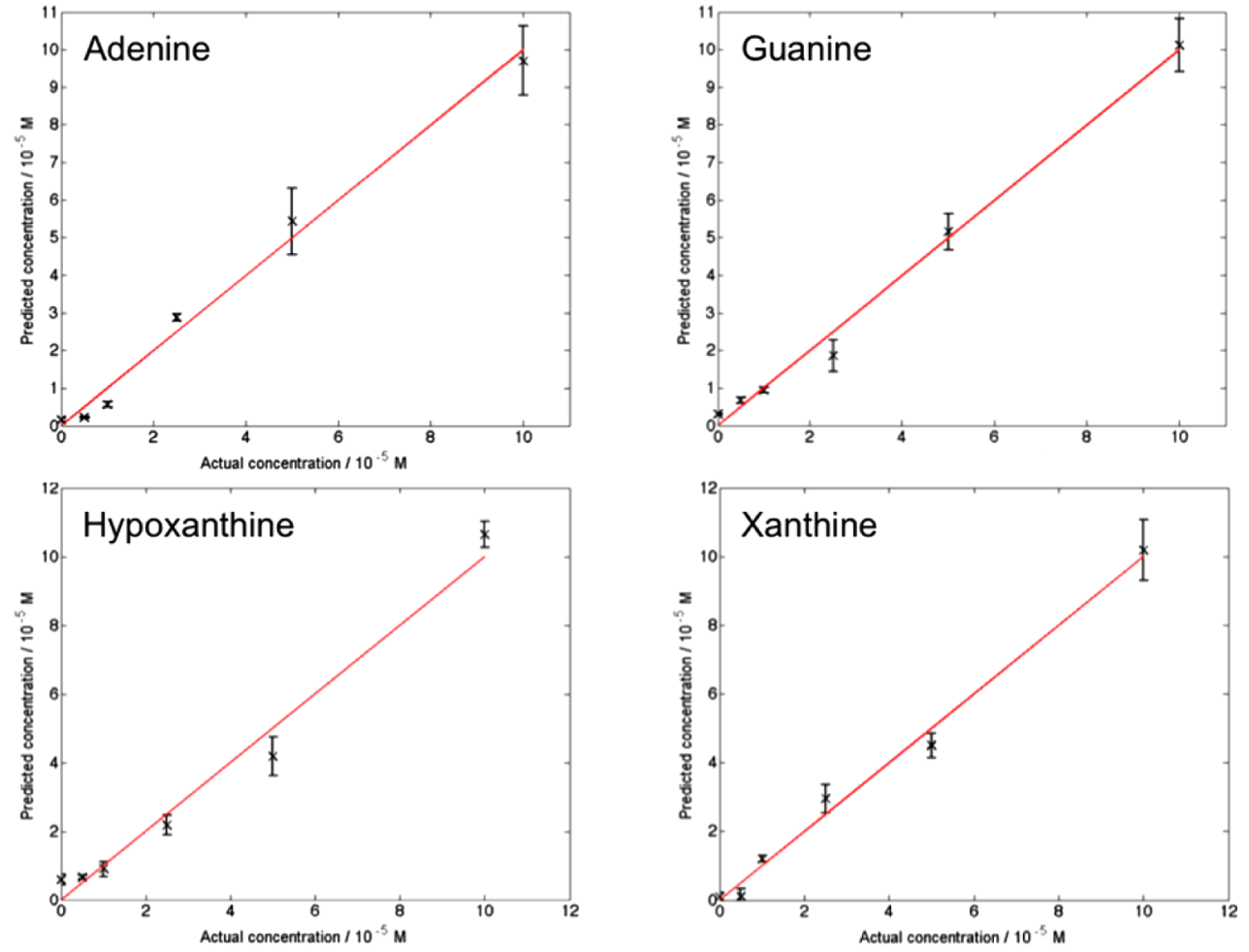

Figure 4. Plots of mean $(n=3)$ predicted concentration against actual concentration for each of the four nucleobases using leave-one-out calibration. All data are from injections of nucleobase mixtures using SERS detection. Error bars show 1 standard deviation. Perfect predictions would lie on the red lines $(y=x$ line $)$.

\section{Table 2. Metrics for HPLC Analysis of Mixtures of Nucleobase Samples Using Simultaneous UV and SERS Detection ${ }^{a}$}

analyte

retention time/s

UV

linearity

LOD (concn)/M

LOD (on column) $/ \mathrm{mol}$

LOQ (concn) $/ \mathrm{M}$

LOQ (on column) $/ \mathrm{mol}$

mean linearity of leave-one out calibration

linearity of leave-one-out predicted vs actual concn

RMS error of leave-one-out predicted vs actual conc.

SERS

peak assignment

linearity

LOD (concn) $/ \mathrm{M}$

LOD (on column) $/ \mathrm{mol}$

LOQ (concn) $/ \mathrm{M}$

$\mathrm{LOQ}$ (on column) $/ \mathrm{mol}$

mean linearity of leave-one- out calibration

linearity of leave-one-out predicted vs actual concn

RMS error of leave-one-out predicted vs actual concn

adenine
268
0.9323
$\left(5 \times 10^{-5}\right)$
$\left(2.5 \times 10^{-9}\right)$
$\mathrm{ND}$
$\mathrm{ND}$
$\mathrm{ND}$
$\mathrm{ND}$
$\mathrm{ND}$
1322
$-\mathrm{N} 7 \mathrm{C} 5+\mathrm{C} 8 \mathrm{~N} 7$ stretch $^{37}$
0.9817
$2.75 \times 10^{-6}$
$1.38 \times 10^{-10}$
$5.91 \times 10^{-6}$
$2.95 \times 10^{-10}$
0.9818
0.9753
$5.51 \times 10^{-6}$

guanine

226

0.9595

$\left(5 \times 10^{-6}\right)$

$\left(2.5 \times 10^{-10}\right)$

ND

ND

ND

ND

ND

669

ring breathing ${ }^{22,37}$

0.9874

$3.45 \times 10^{-6}$

$1.73 \times 10^{-10}$

$5.06 \times 10^{-6}$

$2.53 \times 10^{-10}$

0.9874

0.9838

$4.47 \times 10^{-6}$

.

${ }^{a}$ Overlap of peaks in the UV chromatograms meant that a zero measurement and confident integration of the peaks from certain low-concentration samples was not possible. Therefore, the LOD quoted in brackets is the lowest concentration sample from which a peak could be measured, rather than the statistical LOD. ND = not determinable.

example of the data produced from a mixed sample is shown in Figure 3, and it can be clearly seen how the different SERS band positions can readily be used to quantify coeluting analytes, especially when compared to the simultaneously collected UV absorbance data. Once again, individual calibration plots were made for both the UV and SERS data (Figure S8 in the Supporting Information) to allow comparison; although due to coelution, conclusive identification of each analyte by UV was only possible in the very few cases where the concentration of one analyte was particularly high relative to the other(s). Therefore, accurate quantification from the mixtures by UV was not possible and the LODs quoted represent the lowest sample concentration from which a peak could be measured, rather than the statistical LOD. Leave- 
one-out analysis was also employed for the SERS calibration, with the resulting predictions shown in Figure 4. The full calibration data are reported in Table 2.

Unsurprisingly, the SERS linearity and LOD/LOQ, while very respectable, are not quite as good as for the individual sample injections. While we note this, the difference is by no means extreme and certainly demonstrates that the SERS detection is quantitative in a situation where UV absorbance detection is clearly not possible and a more powerful detector that yields structural information is needed.

In comparison to the at-line fraction collection method, ${ }^{5}$ the LODs and LOQs of the online SERS data from the individual analyte injections are significantly improved for guanine and hypoxanthine and comparable for adenine and xanthine. From the mixed samples, the online SERS LODs and LOQs are only very slightly higher in all cases. The major improvement here comes as the data collection is completed at the same time as the chromatography, rather than requiring chromatography first, followed by lengthy offline SERS analysis using a large Raman microscope. We believe we have demonstrated that our method is far more applicable to high-throughput measurements. Of course Carrillo-Carrión's at-line method allows for far greater control of SERS conditions, particularly in terms of $\mathrm{pH}$, which can significantly affect nanoparticle aggregation and SERS response. ${ }^{5}$ Thus far, this limits our online method to analyte mixtures that are amenable to SERS at similar $\mathrm{pH}$; however, we believe there is the potential for online $\mathrm{pH}$ modification postcolumn where in the future we shall attempt to introduce the buffer in the same manner as the aggregating agent. While this would require analysis-specific validation and for the buffer to be programmed for each analysis, it would allow the online analysis of more complex mixtures using the SERS detection.

Finally in comparison to at-line techniques, our method requires large quantities of colloid. However, as citrate-reduced silver colloid is cheap and straightforward to produce in large quantities, this should not be a significant drawback. In regular use, the syringe driver would easily be replaced with a pump that could handle larger volumes of colloid than the $60 \mathrm{~mL}$ syringes used here, thereby allowing large sample runs to continue unattended for many hours. The online aggregation of the colloid means the waste liquid simply needs to be filtered to remove the silver metal for safe disposal.

\section{CONCLUSION}

We have shown for the first time that online SERS detection for HPLC can be fully quantitative and provide data in real time, simultaneous to other nondestructive online detection techniques, such as UV absorbance. This approach brings with it a significant time saving over at-line methods that require SERS analysis following chromatographic separation and cumbersome fractionation, making this method more applicable to high-throughput applications. The introduction of silver colloid to the postcolumn solvent flow, followed by the introduction of potassium nitrate aggregating agent, gave reproducible SERS spectra from mixtures of purine bases that were able to be quantified even when the analytes were coeluting, based on their unique Raman spectra. The LODs were comparable and sometimes superior to those of current at-line methods, in the region of $100-500 \mathrm{pmol}$ on-column. Our technique does lack the postcolumn control of SERS conditions, such as $\mathrm{pH}$, that previously published at-line methods do, but we believe this problem could be addressed in the future and allow SERS quantification of mixtures of a wide range of analytes.

\section{ASSOCIATED CONTENT}

\section{Supporting Information}

Additional information as noted in text. This material is available free of charge via the Internet at http://pubs.acs.org.

\section{AUTHOR INFORMATION}

\section{Corresponding Author}

*E-mail: roy.goodacre@manchester.ac.uk.

\section{Notes}

The authors declare no competing financial interest.

\section{ACKNOWLEDGMENTS}

D.P.C. would like to thank the BBSRC and Avacta Group plc., in particular Simon Webster and Alastair Smith, for funding this work and his Ph. D. studentship. R.G. thanks U.K. EPSRC and BBSRC and the industrial members of the Bioprocessing Research Industry Club (BRIC) for funding. We would also like to thank Dr. Catherine Winder for the use of the syringe driver.

\section{REFERENCES}

(1) Kuligowski, J.; Quintas, G.; Garrigues, S.; Lendl, B.; de la Guardia, M. TrAC, Trends Anal. Chem. 2010, 29, 544.

(2) Sheng, R.; Ni, F.; Cotton, T. M. Anal. Chem. 1991, 63, 437.

(3) Marquardt, B. J.; Vahey, P. G.; Synovec, R. E.; Burgess, L. W. Anal. Chem. 1999, 71, 4808.

(4) Dijkstra, R. J.; Ariese, F.; Gooijer, C.; Brinkman, U. A. T. TrAC, Trends Anal. Chem. 2005, 24, 304.

(5) Carrillo-Carrión, C.; Armenta, S.; Simonet, B. M.; Valcárcel, M.; Lendl, B. Anal. Chem. 2011, 83, 9391.

(6) Carrillo-Carrion, C.; Simonet, B. M.; Valcarcel, M.; Lendl, B. J. Chromatogr., A 2012, 1225, 55.

(7) Campion, A.; Kambhampati, P. Chem. Soc. Rev. 1998, 27, 241.

(8) Freeman, R. D.; Hammaker, R. M.; Meloan, C. E.; Fateley, W. G. Appl. Spectrosc. 1988, 42, 456.

(9) Cabalin, L. M.; Ruperez, A.; Laserna, J. J. Talanta 1993, 40, 1741.

(10) Somsen, G. W.; Coulter, S. K.; Gooijer, C.; Velthorst, N. H.; Brinkman, U. T. Anal. Chim. Acta 1997, 349, 189.

(11) Sägmüller, B.; Schwarze, B.; Brehm, G.; Trachta, G.; Schneider, S. J. Mol. Struct. 2003, 661-662, 279.

(12) Trachta, G.; Schwarze, B.; Sägmüller, B.; Brehm, G.; Schneider, S. J. Mol. Struct. 2004, 693, 175.

(13) Cabalín, L. M.; Rupérez, A.; Laserna, J. J. Anal. Chim. Acta 1996, 318, 203.

(14) Nissinen, E. Anal. Biochem. 1980, 106, 497.

(15) Ryba, M. J. Chromatogr., A 1981, 219, 245.

(16) Kaneko, K.; Yamanobe, T.; Fujimori, S. Biomed. Chromatogr. 2009, 23, 858.

(17) Huang, Q. H.; Kaiser, K.; Benner, R. Limnol. Oceanogr.: Methods 2012, 10, 608.

(18) Edmonds, C. G.; Vestal, M. L.; McCloskey, J. A. Nucleic Acids Res. 1985, 13, 8197.

(19) Porcelli, B.; Pagani, R.; Lorenzini, L.; De Martino, A.; Catinella, S.; Traldi, P. Rapid Commun. Mass Spectrom. 1994, 8, 443.

(20) Kamel, A. M.; Brown, P. R.; Munson, B. Anal. Chem. 1999, 71, 5481.

(21) Fryčák, P.; Hušková, R.; Adam, T.; Lemr, K. J. Mass Spectrom. 2002, 37, 1242.

(22) Liu, Z.; Li, T.; Wang, E. Analyst 1995, 120, 2181.

(23) Yao, T.; Matsumoto, Y.; Wasa, T. J. Biotechnol. 1990, 14, 89.

(24) Smolenski, R. T.; Lachno, D. R.; Ledingham, S. J. M.; Yacoub, M. H. J. Chromatogr., B: Biomed. Sci. Appl. 1990, 527, 414.

(25) Wynants, J.; Van Belle, H. Anal. Biochem. 1985, 144, 258. 
(26) Lim, K. S.; Huang, S. H.; Jenner, A.; Wang, H. S.; Tang, S. Y.; Halliwell, B. Free Radical Biol. Med. 2006, 40, 1939.

(27) Di Pietro, M. C.; Vannoni, D.; Leoncini, R.; Liso, G.; Guerranti, R.; Marinello, E. J. Chromatogr., B 2001, 751, 87.

(28) Liu, L.; Ouyang, J.; Baeyens, W. R. G. J. Chromatogr., A 2008, 1193, 104.

(29) Lou, S.-N. J. Food Sci. 1998, 63, 442.

(30) Brown, P. R.; Robb, C. S.; Geldart, S. E. J. Chromatogr., A 2002, $965,163$.

(31) del Moral, P. G.; Arin, M. J.; Resines, J. A.; Diez, M. T. J. Chromatogr., B: Anal. Technol. Biomed. Life Sci. 2005, 826, 257.

(32) Chen, H.-J. C.; Chiang, L.-C.; Tseng, M.-C.; Zhang, L. L.; Ni, J.; Chung, F.-L. Chem. Res. Toxicol. 1999, 12, 1119.

(33) Oliveira, E. J.; Watson, D. G. Biomed. Chromatogr. 2000, 14, 351.

(34) Locatelli, M.; Melucci, D.; Carlucci, G.; Locatelli, C. Instrum. Sci. Technol. 2012, 40, 112.

(35) Seger, C.; Sturm, S.; Stuppner, H. Nat. Prod. Rep. 2013, 30, 970.

(36) Exarchou, V.; Krucker, M.; Van Beek, T. A.; Vervoort, J.; Gerothanassis, I. P.; Albert, K. Magn. Reson. Chem. 2005, 43, 681.

(37) Goodacre, R.; Vaidyanathan, S.; Dunn, W. B.; Harrigan, G. G.; Kell, D. B. Trends Biotechnol. 2004, 22, 245.

(38) Lee, P. C.; Meisel, D. J. Phys. Chem. 1982, 86, 3391.

(39) Mabbott, S.; Correa, E.; Cowcher, D. P.; Allwood, J. W.; Goodacre, R. Anal. Chem. 2013, 85, 923.

(40) Cowcher, D. P.; Xu, Y.; Goodacre, R. Anal. Chem. 2013, 85, 3297.

(41) Liu, H.; Dasgupta, P. K. Anal. Chim. Acta 1996, 326, 13.

(42) Quintás, G.; Lendl, B.; Garrigues, S.; de la Guardia, M. J. Chromatogr., A 2008, 1190, 102. 